\title{
Barriers to the diagnosis of affective disorders in Parkinson's Disease
}

\author{
Sara Grace Lutz
}

\section{University of Western Ontario}

In 1817, Dr. James Parkinson ${ }^{1}$ documented the condition of 'shaking palsy,' which was later coined as Parkinson's disease (PD). Parkinson's ${ }^{1}$ description of 'involuntary tremulous motion' and 'lessened muscular power' came to classify the disease into a group of cardinal motor symptoms, particularly tremor, bradykinesia, rigidity, and postural instability. ${ }^{2}$ Parkinson, however, did not consider PD to be a disorder affecting movement alone. Parkinson's ${ }^{1}$ well-known description of one case, '[a] more melancholy object I never beheld,' provided recognition of the affective complications that can occur in PD and demonstrated the level of severity such symptoms can reach.

Despite this early identification of affective symptoms (e.g., depression, mania, anxiety, and panic) as a possible comorbidity of PD, psychiatric disorders often go undiagnosed in clinical practice. This is highlighted in such findings as those by Shulman et al, ${ }^{2}$ whose research revealed that $44 \%$ and $39 \%$ of patients with PD demonstrate clinically significant levels of depression and anxiety, respectively. In this sample of patients, however, only $21 \%$ were identified as having depression, and $19 \%$ as having anxiety, by their treating neurologists. ${ }^{2}$ According to these results, over $50 \%$ of the time, neurologists fail to diagnose affective disorders in their patients. From such findings, it can be argued that affective disorders are a highly prevalent, yet greatly underrecognized, feature of PD. This phenomenon raises the question: why?

Perhaps the answer is that affective disorders in PD are inherently different from those in non-PD populations. This argument stems from the fact that the particular group of symptoms that make up affective disorders in PD tend to be patterned in such a way that is unique to the disease itself. In a comparative analysis of depression among PD and non-PD populations, ${ }^{3}$ it was found that patients with $P D$ tend to have higher levels of dysphoria, pessimism about the future, irritability, sadness, and suicidal ideation.
Furthermore, patients with PD were found to have relatively low levels of guilt, self-blame, and feelings of punishment and failure, which are typically frequent in idiopathic depression. ${ }^{3}$ When looking at the example of anxiety, ${ }^{4}$ patients with PD tend to experience decreased levels of generalized anxiety, which is often suggested to be the most frequent anxiety subtype in the older adult population. ${ }^{5}$ Rather, the most common subtype for patients with PD is that of Anxiety Disorder Not Otherwise Specified (NOS). ${ }^{4}$ In this subtype, anxiety is situational, and patients often experience fluctuating levels of anxiety and panic in direct relation to fluctuations in motor symptoms and effectiveness of parkinsonian medication. ${ }^{4}$

Not only does the symptom presentation of affective disorders in PD tend to differ when compared to non-PD populations, but there also exists a complicated overlap between many of the common symptoms of PD and those of idiopathic affective disorders; this, inevitably, further complicates diagnosis. ${ }^{6-8}$ Shakiness, weakness, and impaired cognition present independently within both PD and anxiety, and a masked-like facial expression in a patient with PD may look identical to the manifestation of anhedonia in depression. ${ }^{8}$

Mere observation of patients in clinical settings may, therefore, lead to under-diagnosis of affective disorders if their presenting symptoms are mistaken for those of PD. Utilization of validated measurement tools for idiopathic affective disorders, however, might also lead to complications. Many of these measurement tools oversample from overlapping symptom domains in the construction of their items. ${ }^{7,8}$ If used with patients with $P D$, the frequency and severity of the affective disorder would be skewed. For example, $78.57 \%$ of the items on the Hamilton Anxiety Rating Scale (HAM-A) ${ }^{9}$ oversample from overlapping symptom domains, asking clinicians to rate such symptoms as muscle tension, tremor of 
hands, increased muscular tone, tendency to sweat, fatigability, insomnia, and weakness. In anxiety scales that possess little oversampling from these domains, such as the State Trait Anxiety Inventory (STAI), ${ }^{10}$ items tend to be focused entirely on generalized anxiety, which is less frequent in PD. It is, therefore, clear that such measurement tools cannot be validly used with patients with PD and that the construct of affective disorders itself is inherently different in the PD population.

Perhaps a more valid approach to diagnosing affective disorders in PD is to generate measurement tools that assess facets of affective disorders that are independent of the symptoms of PD. In order to achieve this goal, future research must aim to operationalize the construct of the affective disorder under study, thereby creating an operational definition that pertains directly to individuals with PD. It is only after PD-specific affective disorders are operationalized that respective measurement tools can be generated. Once this is achieved, it can be hoped that affective disorders will be properly identified in health care settings, and, with this improved rate of diagnosis, subsequent improvements in treatment can evolve.

\section{References}

1. Parkinson J. An essay on the shaking palsy. London: Whittingham and Rowland; 1817.

2. Fahn S. Description of Parkinson's disease as a clinical syndrome. Ann N Y Acad. Sci. 2003; 991:1-14.

3. Brown RG, MacCarthy B, Gotham AM, Der GJ, Marsden CD. Depression and disability in Parkinson's disease: A follow-up of 132 cases. Psychol Med. 1988; 18(1):49-55.

4. Pontone GM, Williams JR, Anderson KE, Chase G, Goldstein SA, Grill S et al. Prevalence of anxiety disorders and anxiety subtypes in patients with Parkinson's disease. Mov Disord. 2009; 24(9):1333-8.

5. Wolitzky-Taylor KB, Castriotta N, Lenze EJ, Stanley MA, Craske MG. Anxiety disorders in older adults: A comprehensive review. Depress Anxiety. 2010; 27(2):190-211.

6. Bonnet AM, Jutras MF, Czernecki V, Corvol JC, Vidailhet M. Nonmotor symptoms in Parkinson's disease in 2012: Relevant clinical aspects. Adv Neurol. 2012; 2012:1-15.

7. Higginson $\mathrm{Cl}$, Fields JA, Koller WC, Troster Al. Questionnaire assessment potentially overestimates anxiety in Parkinson's disease. J Clin Psychol Med S. $2001 ; 8(2): 95-9$.

8. Shrag A, Barone P, Brown RG et al. Depression rating scales in Parkinson's disease: Critique and recommendations. Mov Disord. 2007; 22:1077-1092.

9. Hamilton M. The assessment of anxiety states by rating. Brit J Med Psychol. 1959; 32:50-55

10. Spielberger $C D$, Gorsuch RL, Lushene PR, Vagg PR, Jacobs AG. Manual for the State-Trait Anxiety Inventory. Palo Alto, CA: Consulting Psychologists Press, Inc; 1983.

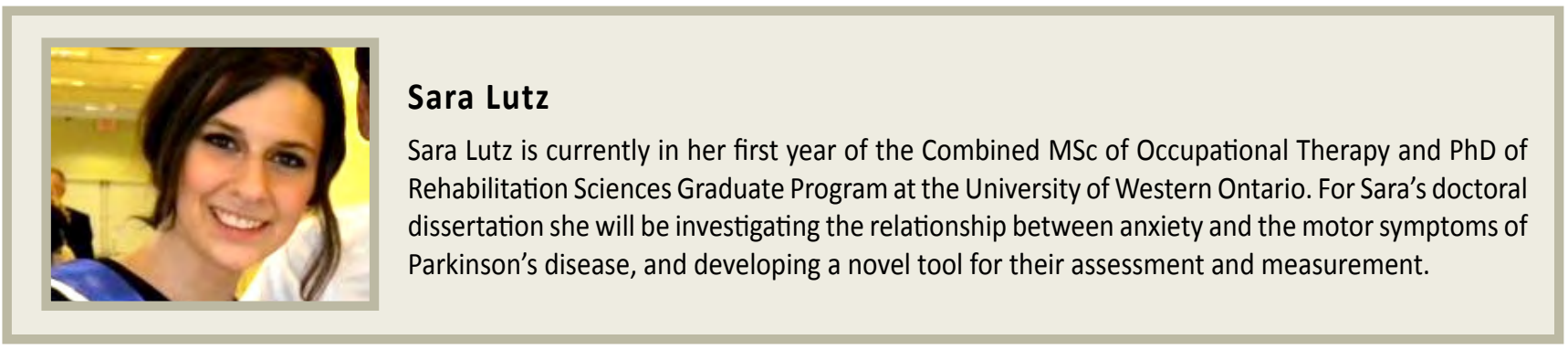

